\title{
On Avoidance: Reflections on Processes of Socio-spatial Structuring
}

\author{
Daniel Koch \\ KTH School of Architecture, Royal Institute of Technology, Sweden
}

Copyright $\subset 2016$ by authors, all rights reserved. Authors agree that this article remains permanently open access under the terms of the Creative Commons Attribution License 4.0 International License

\begin{abstract}
This essay is a reflection on how the 'social' comes into being, and how it relates to questions of architecture and urban environments. Taking its departure from a statement in The Social Logic of Space, where it is said spatial configuration affects social relations in how it structures patterns of movement, encounter and avoidance, it develops on the notion of avoidance as a socially and spatially structuring behaviour. One reason for such a focus is a contemporary focus in urban and architectural discourse of encounters and co-presence, whereas patterns and actions of avoidance are less often present. On the one hand, it argues that studying of how space generates, allows, or prevents patterns of avoidance is a missing key question that may also further develop discourses of patterns of encounter. On the other hand, it is suggested that a focus on avoidance demands a series of questions to be answered that is beneficial for understanding socio-spatial behaviour and structures in general. These questions concern the temporal, cultural, personal, and spatial embeddedness of actions that includes memory and myth as well as projection and imagination. Avoidance, furthermore, is argued to be as social an action as encounters, with considerable direct and indirect structuring effects, and thereby to constitute an important piece in socio-spatial structuring processes.
\end{abstract}

Keywords Spatial Configuration, Performativity, Avoidance, Encounter Patterns, Social Structuring, Spatial Behaviour, Architecture Theory

\section{Introduction}

This essay is a reflection on how the 'social' comes into being, and how it relates to questions of architecture and urban environments. It does not intend to give an answer to what 'the social' is, as much as introduce and discuss a few aspects thereof. My main point of interest is aspects of how 'social structures' come to be in relation to space. In a sense, it is a response to recent tendencies in treatment of 'the social', 'urban life', or social structures in theory as well as practice to become somewhat translatable either to Georg Simmel's sociability, Guy DeBord's spectacle, or a locally situated performativity. ${ }^{1}$ I find it somewhat troubling when such approaches are too readily combined under the assumption that this particular form of enacted sociality is good, carrying implications that other ways of acting are $\mathrm{bad}$. The discussion will operate both directly in highlighting these aspects, and illustratively through the concrete question outlining a kind of 'social' that is in need of being reincorporated more thoroughly into both theory and practice.

The point of departure is the first paragraph of the preface of The Social Logic of Space, where Hillier and Hanson [1] discuss how architecture structures the space in which we live, and that "[i]n that it does so, it has a direct relation - rather than a merely symbolic one - to social life, since it provides the material preconditions for the patterns of movement, encounter and avoidance which are the material realisation - as well as sometimes the generator of social relations." (ix) While the discussion here pertains to a wider set of theories and practices, it serves well to raise some questions regarding our theorisation of society and space. Over the following decades, research in the field 'space syntax' at large has increasingly focused on the interrelations between spatial configuration and movement, on the one hand, and various forms of encounters (co-presence, face-to-face interaction, physical encounters), on the other; a development that is paralleled in several fields of inquiry. My intention in this essay is to develop the third term in the quote: that of avoidance. I intend to show how avoidance demands that a series of questions are

1 This focus seems to partially stem from methodology; co-presence and interaction can be observed, as can certain definitions of 'liveliness', and locations of individuals and events can be recorded in various ways. In more local settings whom interacts with whom when can be documented, in mass observations the where, how much, and how many of interactions. Conversely, it is a more difficult process to find where people chose not to go and why. In some cases, it is a theoretical principle, such as (in a somewhat simplistic interpretation of) Latour's [2] claims that the important thing is the networks, assemblages, and interactions, not necessarily who or what is interacting, or regarding what. 
addressed, promoting a more complex understanding of the 'social' and 'cultural', and how these factors play out between individuals, space, and society in ways that are not necessarily accounted for when we maintain a focus on 'encounters' or 'interactions'. ${ }^{2}$ This is not because studying social interactions, communities, and co-presences has been unfruitful; it is rather to say that the extended implications and socially structuring effects of 'avoidance' have not been addressed to the extent they deserve.

The discussion aims to address how avoidance actions operate through spatial configuration and hand how certain architectural or spatial features can be understood as mechanics of avoidance. As this essay is a first step in addressing these issues I will focus on drawing the larger picture, well aware that this will give only a partial account leaving some aspects unaddressed and some touched on only briefly. For this reason, this essay will operate on a theoretical, discursive level, inter-punctuated by empirical support. It will do so through a series of thematic discussions intended to make sense of avoidance as a socio-culturally structuring and tactical behaviour.

Studying avoidance phenomena in architectural and urban studies, there are three categories of research papers that tend to dominate literature searches: research on (fear of) sexual assault; behaviour of criminals; and overall safety and security questions in urban environments including CPTED [3-10]. While this will not be the focus of my discussion, it must be acknowledged, including the disturbing implications of the first being the most common by far. Set in relation to the emphasis on research on encounters, this taken together risks painting the picture that avoidance in general is a problem or a symptom, and that encounters, co-presence and interactions are a kind of cure.

While dealing with things that have purchase on these situations, I will instead focus on avoidance as a commonplace part of everyday behaviour that has both positive and negative intents and effects, and how it participates in social structuring. To do this, avoidance must be studied as a complex social, spatial, and strategic-tactical phenomenon, that can also be seen as empowering tactics and strategies of resistance or freedom $[11,12]$, which at times is that which allows for vastly different (sub)cultures to inhabit dense spatial structures such as cities [13,14], or to, for example, create situations "wherein young people learn to think outside the box, or at least learn to think inside a different box" [15] (p. 91). ${ }^{3}$ While I will be

2 I use 'we' and 'I' here deliberately while recognizing it can be problematic. Throughout the paper I use 'we' where I am reasoning around generalized phenomena and developing on thoughts, to differ from where I present empirical facts or results of research, and in order to place me ('I') as active part of this discussion on its general level, and to differ it from descriptions of 'other' (one, they, you) placing me as a researcher outside the discussion. 3 An area where avoidance has been studied, albeit differently from in this essay, is in studies of conditions under which various social or identity groups escape oppression, empower themselves, or find breaths of freedom by literally, explicitly or subtly creating heterotopias outside of hegemonic control. Here the considerable work of queer and feminist studies in architecture certainly demonstrates a wide range of potentials as well as problems with avoidance, productions of heterotopias, and other similar tactics (e.g the works of Bonnevier [16]). making use of tactics and strategies largely based on the writings of Michel De Certeau [17] I am not focusing on the type of avoidance tactics he presents, such as for instance, shifting to the other side of the street to avoid an unwanted encounter, or similar discussions of Goffman's [18] 'civil inattention' concerning how people negotiate behaviour in public spaces to avoid direct communication while maintaining a civil presence of oftentimes conflicting identities or actions, but a behaviour that extends such actions both temporally and spatially. ${ }^{4}$ In addition, in this discussion, I will draw the writings of De Certeau as well as that of Augoyard [20], Barthes [21], Ricoeur [22], Rose [12] and Butler [23].

To introduce the more complex understanding of 'spatial' avoidance that I will discuss, I will return to The Social Logic of Space [1] where they use the example of children playing hide-and-seek to introduce how children can read a spatial configuarition and assess its capability to accommodate the game (p. 37-38). This example can be developed looking more closely at the practice of hide and seek. That is, how hiding depends on an understanding of seeking and vice versa. Hiding, to put it simply, is best done where the seeker is least likely to look, and finding best where the one hiding has hidden, recursively dependent on expectations on the seeker. That is, the seeker is asked to respond by negating the behaviour that is expected of her or him by the ones who are hiding. In more complex forms, such as that of Burken (or Kurragömma med Dunk) in Sweden, or Oro as played by Igbo children in Nigeria, these activities become dynamic.

Both variants of hide and seek activates the one who hides as well as the seeker and have similar characteristics. In Burken, the seeker has a home base, burken, which has two roles: when having found someone, the seeker has to return to the home base and touch it (usually kicking it) and say the name and location of the person found. It can, however, also save the ones hiding: if one of those hiding reaches it unfound, s/he becomes safe and has 'won'. The game is then played either until everyone is found or safe. ${ }^{5}$ In Oro, the hide-and-seek is combined with a game of tag, so the seeker needs to chase and tag the ones $\mathrm{s} / \mathrm{he}$ finds, and the hider becomes safe on reaching the home base. Both variants set an intricate tactical social game in motion. Not only do 'I' need to remain hidden, but also I need to be on the move, while avoiding the seeker. I need to weigh the risks of being discovered against the chance of winning, and

4 Goffman's 'civil inattention', which has been criticised for looking too neutrally at something that is oftentimes a power play and disciplinary behaviour, is largely based on discussing how people interact through knowledge and learnt as well as constantly negotiated behaviour in each situation, pointing to how apparent non-interaction, in fact, is dependent on constant communication in various low key, nonverbal forms. While Goffman does not explicitly speak of avoidance, his work is important for what Lofland [19] develops more directly as 'avoidance'.

5 In some variants, if any of the ones hiding reaches the home base everyone is 'safe' and the game is over with the seeker losing. This introduces further complexity beyond that it extends the time of the game, but also alters the type of game it is: the difference between the ones hiding operating as a team or as competing individuals have significant impact on what kind of sociality is performed through it. 
I need to best possible anticipate the actions of the seeker so as to know best when and how to move - which is when neither can see one another. Similarly the seeker needs to take into consideration the tactics of the ones hiding for reaching the base or retreating into hiding in a spiral of actors trying to out-predict each other. That is, the interaction here is both on a material, observable level, and on a mental, tactical level. While it can be argued that children's games serve to train and develop various social skills [24], the intention is not to claim hide-and-seek as a model for society but to exemplify a tactical level of socio-spatial interaction that has implications for how we understand, analyse, and work with 'the social logic of space'.

\section{Programmed Avoidance: Courthouses, Homes, and Fashion Retail}

An initial discussion on 'avoidance' and architecture may most easily pass through how patterns of encounters and avoidance are regulated in buildings. Studies of prisons, schools, hospitals and museums, for instance, have shown how these institutions tend to strictly separate and differentiate the range of freedom and access for different actors for many different reasons [1, 25-28]. Julienne Hanson's [29] study of courthouses becomes a particularly clear example, investigating a building type that is conditioned by keeping different groups strictly separate throughout the whole building except in a specific room where they should all be able to meet. While ostensibly dependent on functional or practical needs, the mentioned typologies are also performing socio-cultural conditioning, not the least captured in Markus' [28] labelling of them as spaces of formation and reformation, drawing on discussions by Foucault [25]. In Decoding Homes and Houses, Hanson develops on this, perhaps most explicitly in how "[t]hough well integrated, the lobbies, passages and corridors of Coleshill are designed to institutionalise avoidance." [30] (p. 193) She continues to describe how this is done through how

$[\mathrm{m}]$ ovement within the interior, particularly the domestic routines of the servants, is programmed by rules governing conduct which ensure that spaces designed for efficient movement remain empty for most of the time. Activities are assigned to rooms which by means of their local and global configurational characteristics, rationally differentiate service functions from those which are served and common areas from private spaces. (p. 193)

Hanson here identifies existing practice of what was proposed as the ideal by Alexander Klein in 1928 (Figure 1), showing 'bad' and 'good' plan solutions blending or separating flows of family and servants (c.f. [31, 32]).
Steadman [33] gives another interesting example in differentiating the homes of British and French housing traditions in the 19th and early 20th century, where the former mostly had one exterior entry per apartment and the latter one entry to a common staircase. This, according to Steadman, because it was awkward in the British context to meet strangers - even neighbours - inside the domain delimited by the exterior entrance, and downright unthinkable to meet someone else's servants there, whereas in the French tradition this was commonplace and culturally unproblematic. ${ }^{6}$ Avoidance seems simply to be a crucial part of the social and cultural practice of architecture in general through how it materially regulates ranges of access and separates the included from the excluded.

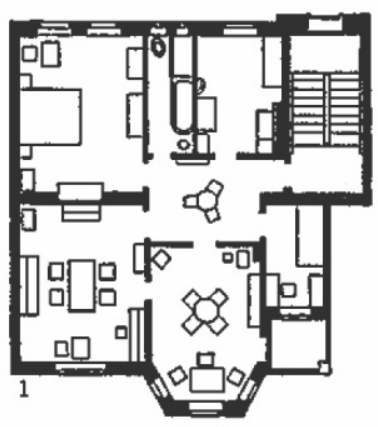

A. Bad Example

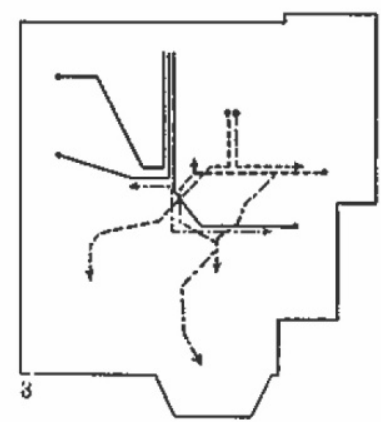

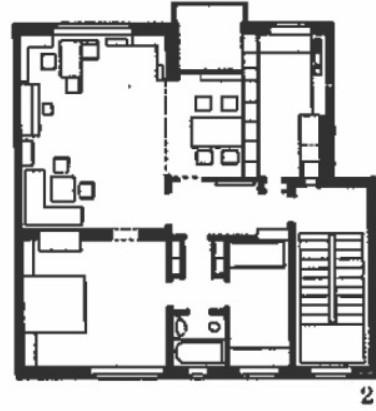

B. Good Example

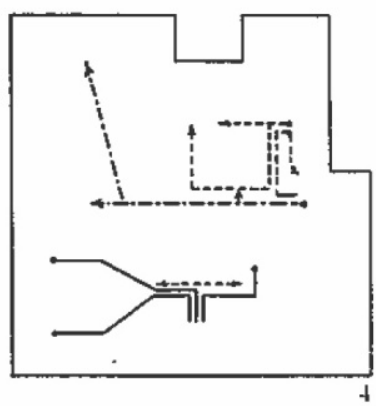

Figure 1. Alexander Klein, the functional house for frictionless living, 1928, originally in Bauer, Catherine, Modern Housing, 1934, p. 203.

A more implicit case of programmed avoidance can be found in the Swedish department store Åhlens City [35]. As an example, the two identity branches of 'tailored fashion' and 'street fashion' on the men's fashion floor - itself separated out by biological sex and placed deep in the building to ensure it is less likely to be visited by 'others' here both reach out from close to the main set of stairs.

6 Steadman develops further on control and ownership patterns, as well as the relation to a building 'as such' differing between the cultures, so that in the British context the house is the important entity of private (family) ownership and control, whereas a corresponding unit would rather be the apartment in the French tradition. This is a note in line with White hand and Carr's [34] observation on the differentiated traditions and ideals for homes between what they label 'British' and 'Continental' contexts (the latter largely seen as France, Italy and Spain), also pointing to the comparative importance of owning a 'house' with its own entry and preferably garden in the former partially related to a tradition of nobility owning country estates, whereas palaces in cities were more common in the latter. 
However, rather quickly the two become obscured from one another, and the further into them - the higher up their internal fashion hierarchy one moves - the more visually separated they are, and the less likely one is to see or encounter someone or something related to one when shopping in the other (as demonstrated in Figure 2). Flows of customers and sequences of actions are tailored to avoid one another based on positions in fashion hierarchies and identity categories. In Hanson's [30] terms, they are insulated from one another through intermediate spaces, and they are sequenced so that it is clear that one is distinctly separated from the other not only locally, but in their global configurational relations.
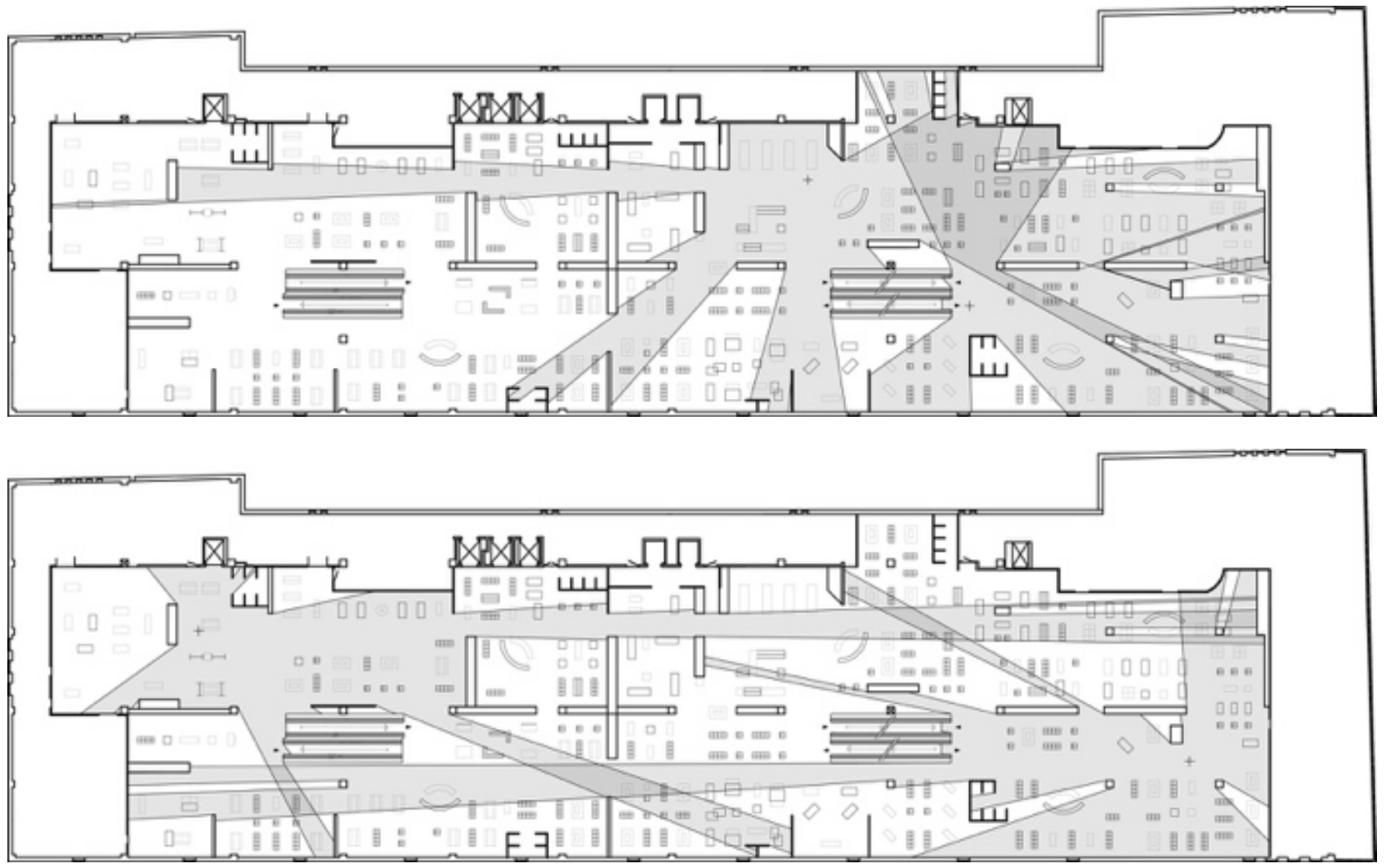

Figure 2. The two branches of fashion in Åhlens City are located so as to maximize the differentiation and ensure that customers shopping will either avoid each other (in the context of shopping in those branches). Isovists from encountering the branch moving from the main staircase (top) and endpoints / highest hierarchy brand (bottom). ${ }^{7}$

7 Isovists are developed from viewsheds and form two-dimensional extentions of uninterrupted space from a point; often, isovists are used to investigate visibility properties of individual locations or relations of visibilities from a range of positions [36, 37, 38]. 
On the level of the city it is for example common to avoid meetings between children and heavy car traffic. Similarly, arrangements are often made both through spatial and temporal planning to avoid conflict between large-scale delivery and pedestrian traffic. Shops may locate deliberately in more or less off-center locations [39]. Encounters with functions, land uses, and many other things are negotiated to foster or avoid everyday encounters with them or between them (e.g. [11, 40, 41]). These differentiations can operate directly - keeping things located separately - or indirectly - such as structuring whether travel routes to and from them overlap, or whether one is located along common routes to and from another or not. While interlinked in effects, the reasons for these programmed avoidances operate both in how the city is lived functionally, and how it acts in the generation of meanings and identities.

It must be noted here that the examples given are historically and culturally situated. Degrees of control, separation and surveillance vary over time and space also within ostensibly the same use-types of buildings, as well as the modes through which they are performed [25]. The typologies themselves also have their social, historical and cultural specificity - the 'use-types' referenced have not always existed, nor are they defined similarly from social or functional perspectives in different societies today $[42,43]$. Similarly, what a 'street' or a 'square' is differs between contexts. This needs to be recognised in the coming discussion and its examples need to be considered as a way to tease out principles, where enactment will take different forms depending on who performs it and in what context. However, while 'programmed' avoidance forms one important ground for the coming - forming referents and contexts to relate to and conditions structuring actions, allowing, supporting or even institutionalising avoidance through configuration and socio-structural performativity of avoidance tactics - the main focus of the discussion here is on a more elusive form of avoidance.

\section{Performed Avoidance}

If 'programmed avoidance' is a way to discuss how avoidance is materialised, arranged or institutionalised to certain degrees, another side of the question concerns individual and collective action, and how this structures social relations. That is, avoidance as an act that is part of society as a performative system $(23,12)$. This is the central concern of the discussion in this essay. In a performative understanding, social structures are neither pre-existent nor false, but exist in that they are enacted either through the mechanics that generate them, or as related to in conforming and resisting acts. In effect, while performative systems can appear stable, they are always under (re)construction through their own enactment, where the enactment is not of the system as such but through the myriad of actions of the people living it. As Rose [12] notes, "[t]his is the uniqueness of performative systems: they rely on instability, elaboration, and difference, rather than stability, consistency, and routine." (p. 393) ${ }^{8}$

At the same time, it is important that performative structures are recognised as structures, even if their nature is understood as retained and generated through performance, and that they can be recognised and, once recognised, incorporated into actions that both maintain and challenge them. Rose [12] further develops regarding resistance geographies how it would be faulty to assume that there is one dominant hegemony operating on its own terms, pre-existing actions and reactions, and a completely other order to resist it. Rather, they must be considered as part of the same system. That is, resistance can be part in maintaining the structure. However, while such structures are not 'pre-existing', this does not remove the temporality of social relations where earlier experience affects the here and now as well as the future [45]. Avoidance actions thereby both challenge and reaffirm the 'current state', but there is need to further investigate this relation.

\section{Pattern Recognition}

An important point of departure for the discussion is that in the midst of the complexity that forms cities, there are certain aspects of the way they are inhabited that form reoccurring patterns. I am not here interested in the mechanics as much as the simple observation of recurrent patterns as part of the structuring conditions of avoidance depends on the ability we have to recognise and construct an understanding of such patterns out of instances and series of situations (or perhaps better out of the continuity of daily life); that is, our ability to extract a configuration from a sequence [22]. Through our navigations of the city we learn of where there are likely to be many or few people at what times of the day, and general ideas of what goes on where through direct experience or inference.

This capacity of people and societies to recognise and act in relation to larger scale phenomena is important to stress in a context with increasing focus on the interactions of individuals and their specific locale and context. As noted, this is not contrary to a performative understanding but requires that we address past experiences and emergent collective behaviours as part thereof. The role this plays in everyday action is further developed by Vinicius Netto [46] who explains that "[w]e join a social situation by knowing it is held (or knowing that it is possibly or probably held) in a certain place; we 'refer' our practices and ourselves to that place, being guided by its meaning, understanding its social content as a context of particular acts." (p. 374) We act in and through space based on our knowledge of social actions in space. A more concrete, illustrative example I have made

8 Rose here, arguably, takes Gramsci's [44] notion of hegemony as a process that is always negotiable and always in becoming further by reducing or even removing differences between hegemonic and resistance structures, where Gramsci makes more of a difference between the power operations to ensure stability and the tactics of subversion or social change. 
use of earlier [35] is how the first time the protagonist and Nadja go out to eat in the novel of the same name by André Breton [47], the two choose to go to a part of the city where they expect to experience a suitable, calm pace but also where they expect to only encounter strangers - it is crucial for their activity to not run into friends or family.

Together with programmed avoidance, this sets up a complex and layered relation between society and space, or space and behaviour, action, use, or any other term for the 'social' that is employed; while individual perception of space remains an important consideration, recognition of the habits and actions of others must be taken into account. In this, it is important to recognise that pattern recognition as discussed here is predicated upon abstractions, generalisations, and recombination of sequences and events into configurations and tendencies, which tend to introduce distortions.

\section{Of 'Us' and 'the Other'}

Taking many different forms, this 'pattern recognition' translates into knowledges of specific places, of individual others, of groups of characters of others, and of the generic 'Other' (c.f. [23]), as well as types, for lack of a better word, of places and situations both locally and in their context. The constructed knowledge, partially organised as principles or experiences, is something we can relate to as we act in a made-sense-of city, a sense saturated by both 'spatial' and 'social' characteristics to which one must relate in order to avoid. In particular, in order to avoid, as the two protagonists in the novel Nadja discover, it is necessary to relate to the actions and habits of others and the Other set in relation to intentions and goals of one's own.

When it comes to our knowledge of others, it can be argued that this follows similar processes to that of knowledge of the city. As we get to know each other, we learn codes of behaviour and expressions and recognise subtle differences, including perceiving 'us' as diverse, complex, differentiated, and individual [48]. ${ }^{9}$ Conversely, the less we know someone the more we need to depend on what was learnt the few times we have encountered them, and on assumptions extrapolated from what we have heard about them, when we have met someone similar to them in whichever respect we find similarity, or what we have heard about someone similar to them. While 'we' are complex, individualized, and differentiated, 'others' are grouped, categorised, and equalized into either generic 'others', or perhaps more commonly, 'types' of others, leaving aside,

\footnotetext{
9 Introducing Bourdieu in the discussion can be problematic as it brings in a wide set of theories and research that can conflict with some of the arguments made here, but it is here done because of the argument in Distinction that social structuring and the 'membership' of groups is based on our knowledge of the codes and practices of that group rather than active inclusion and exclusion; conversely, others, or other groups, will be perceived as similar to the point of homogeneity since we do not know their codes of differentiation. Thus there are two differentiating processes at work: between groups (classes in Bourideu's terms), and between individuals within the groups, where the knowledge of codes and tastes is what forms the group as an 'us'.
}

for now, the relations to the Other. ${ }^{10}$

This further leads to processes of Othering (e.g. [23, 25, $49,50]$ ); individuals or groups being constructed as Other primarily, further strengthened by selective readings of codes and behaviours. Recognition of anything different from 'us' or 'me' may take precedence over any similarities, and actions and relations may stress and strengthen this otherness as well as introduce expectations on these others to be Other.

This is important to note as, arguably, our perceptions of what others may do or intend often depends on generalized understandings of person 'types' and a complex and contradictory construct of 'us' and 'others' at times equalising the Other with 'me' ('I would do it like this, so they will too'), at times distancing the Other from 'me' ('unlike me, they would do it like this'). At any point we act in relation to 'others', therefore, especially specific groups or individuals, this becomes a necessary component of the act.

\section{Memory, Mythology, Metonymy}

If the above forms parts of how cities are made sense of (c.f. [46]), and how this sense is crucial for avoidance actions, it is worth placing a stress on the term 'made'. This is because making sense of something is an act; it is something done, and done differently by different individuals as well as collectives (groups, subcultures, cultures, societies) in different situations. For this discussion, I prefer to operate primarily in relation to the hermeneutic process as described by Paul Ricoeur [22]. For Ricoeur, the interpretation process first goes through constructing the world in front of the work based on the clues given by 'the work', complemented by assumptions, knowledge, understandings, and preconceptions of the subject in a semi-logical process. It is from within and in relation to this world the reader produces meaning, and meaning is in constant negotiation as the world in front of the work is always under reconstruction. As a result, there are only negotiated meanings: inconsistencies and contradictions generated by the abstraction and generalisation processes of, for instance, 'pattern recognition' and processes of subjectification and Othering as discussed above, are constantly renegotiated to provide a reasonably coherent World in relation to which meanings can be made in the situation in which the 'reader' acts.

This 'World' is always fundamentally imperfect, piecemeal and assembled. No matter how well we know any specific person or what knowledge we have of groups and their habits, we do not know the full, saturated

$10 \mathrm{It}$ is important here to point out that rather than a strict typology, the kind of 'type' in action here is one that is situationally formed and configured (whether for example 'elderly', 'dressed in suit', 'muscular', or any other characteristics true or false is primary categorising operation may alter from situation to situation), and imperfectly formed from our selective readings and interpretations of a wide range of possible features to take into account. 
complexity of them as individuals or groups, nor do we usually know their specific locations, actions, and behaviours when we are not around them. In most cases, furthermore, the knowledge is constructed from a range of sources that provide anything from concrete information to loosely formulated assumptions or prejudices, with widely different sources. For this reason, I choose to take the discussion further through the filters of memory, mythology, and metonymy (c.f. [17, 20, 21, 51, 52]), as I believe these filters provide a good set through which to understand 'avoidance' and its relation to socio-cultural structuring processes.

Memory here means knowledge constructed through personal experience. While not providing 'truth', it forms a direct source of reference. It can be regarding either individuals, groups, people in general, or places, all depending on degrees of abstractions, categorisations, and projections. As Ferguson notes [45], memory is also always contemporary, in that it is the concurrent way in which past events are recollected and made sense of, rather than past events themselves. At the same time, it has both direct and indirect purchase on behaviour [53]. As a concrete example, Augoyard [20] discusses how an elderly woman prefers not to go through the suburb's centre because she has experienced that certain groups of youths tend to hang out there. Her memory here stops at 'often when I have visited the centre there have been people who appear to be the same group of youths there', but this is extended through interpretations of their behaviour and assumptions of their mindsets and intents. They are 'othered', and othered as dangerous, and hence to be avoided.

Furthermore 'memory' works well since it highlights the ambiguous relation to empirical fact in that it can change over time, because what one remembers and how one remembers it is related to what one has experienced before, as well as affecting future memory making (c.f. [51, 54]). Nick Kaye [52] here provides an illustrative example of Michelangelo Pistoletto's work Le Stanze (the rooms) ${ }^{11}$, "[e]xposing the conditions in and under which a work is always in the process of being anticipated, produced and remembered, where 'real space' is written over by anticipation and imagination." (p. 31). Amongst many other things, this series of artworks show how the reception and interpretation of any work depends on both memory and projection - that is, what one has experienced before, what expectations one has - and how later experiences potentially can rewrite the meaning of earlier. Memory here is demonstrated as an active faculty in itself, operating both for the purposes of futural projection and for interpretation.

Mythology, in comparison, stands for knowledge gained from others regarding yet others. The reason to use 'mythology' is due to how 'myth' always stands in an

11 Le Stanze (the rooms) was a work consisting of twelve consecutive installations, each consisting of a mirror artwork placed in one of the door openings of a movement axis of the Christian Stein Gallery in Turin from October 1975 to September 1976. unclear relation to the degree of 'truth' which it contains, and the inability to discern from the myth itself the degree to which it is true due to this nature [21]. The myth provides meaningful information regardless of its truth; it does not matter whether it is true that a certain person or group does one thing or the other - the impact of the myth depends on how it translates into meaningful information that is related to in both discourse and action.

Many of the myths I refer to here are things 'people know', without being fully able to say how or where they learnt it from; for instance, societal myths that are propagated by most everyone, often without clear origins or intentions come to be established as 'social knowledge'. Lofland [55] gives the example of how discourses of danger and especially sexual assault can be seen as a form of disciplining of (young) women as much as, or even more than ensuring their safe-keeping. ${ }^{12}$ But I also include under the category of mythology a range of more directly second-hand information having a similar character due to being imprecise - if only simply because of how retelling is unable to give a full account of the complexity of a situation or the sequences of events leading to and following from an event. ${ }^{13}$ Filtering the discussion through mythology allows us to study the effects of the myths rather than the 'truthfulness' or sources of what is being said, or the intents behind their propagation. ${ }^{14}$

This reasoning is again similar to Netto's [46] in how events and places extend beyond themselves through various forms of communication, such as through material and immaterial traces, when events are communicated at another place or another time, or through media communication of different kinds, and that this may take part in how they are incorporated in the unfolding of everyday activity.

Metonymy, finally, is a process of substitution by means of contiguity. That is, that something is allowed to stand in for something else by means of representational reference, such as 'Wall Street' can stand for the U.S. financial and corporate sector. The reason why this is of interest is because, arguably, the metonymy may serve as the referent as much as that to which it refers when we speak of mythology or sense-making. That is, a 'dark tunnel' can come to stand for danger not because it is dangerous as such, but because it holds a metonymic relationship to a range of

12 It should be noted that Lofland questions the extent to which the discourse affects actual behaviour, although she does it primarily by pointing out that the evidence for either case was lacking. While not unambiguously, others have shown effects on behaviour to be real $[3,20$, 53]. It is worth to stress that effects depend on both contextual (where, when, in what location, concerning what) and individual (who) factors as well as what discourse is considered.

13 I want to stress I am not saying people are not truthful or that they are intentionally misleading - the transformation into myths is based on the imperfection of memory and communication, as well as the aforementioned inability for anyone to grasp the full complexity of any individual situation or person.

14 This is not to say that studying the basis, generation and propagation intents of myths is not of equal importance, it is rather to say that for the discussion of this essay it is the effects of myths that are of interest, and it is specifically that myths in this sense have purchase on action regardless of truth that makes the term productive. 
acts which one might wish to avoid, or a playground could come to stand for children, play, or happiness dependent on memories, myths and experiences. Metonymy becomes important in processes of othering, as it can be used to describe how certain individuals, acts, or behaviours come to stand for a group, for others, for the generic other, or for the Other. ${ }^{15}$

Arguably, the most consolidated empirical support for these figures comes from the considerable work of Jean-Francois Augoyard in Step by Step: Everyday walks in a French urban housing project [20]. In his investigations of a French suburb he shows how the inhabitants relate to their environment through a set of ideas that can be largely summarized as the above, although he differentiates further. He clearly demonstrates how people relate to 'figures' rather than to 'facts' as they decide what to do and how to go about doing it. ${ }^{16}$ In reoccurring sequences they relate to imperfect, simplified, abstracted, or generalized figures, sometimes in the form of concrete places as representative of these, and at other times in relation to specific understandings of others or the Other, which is what has been developed above as memory, myth and metonymy on the one hand, and as the world in front of the work, on the other, as an intermediate between the concrete, material 'real' and the situated act of individual or collective meaning-making. It is, however, important to keep in mind that conscious referral to such figures is only one piece of the puzzle, and that subconscious or affective effects need also to be considered [53] even though there is not space currently to further elaborate.

\section{Again, Avoidance}

To reiterate, avoidance is an everyday part of life that can take many different forms, which vary through history and between cultures. It is arranged spatially for both political and pragmatic, functional reasons. Simmel [13] and Benjamin [14] argue certain forms of avoidance are necessary components of modern urban life - such as the capacity to be in a crowd while retaining a certain privacy, which is also part of an urban 'social contract'. This extends into subtle, non-verbal communication that allows people to meet with little recognition of one another but avoid collisions or awkward situations as discussed by Goffman [18], or changing sides of streets or choosing an alternate

15 It may be of interest here to relate to Slavoj Žižek's The Pervert's Guide to Ideology [56], where he fairly extensively discusses how fear tends to take this kind of form - that is, fears are allowed to materialize and coalesce into specific figures such that the shark in Jaws can stand for a wide range of unrelated fears for many different people. On one hand, Žižek discusses how something can symbolically represent something else that is radically different, but on the other hand, he specifically discusses how large, complex issues are allowed to be simplified to smaller, simpler, and crucially, to identifiable things.

16 It may be in place here to reiterate the independent relation between 'figure' and 'fact'. Independent relation should be understood literally as independent and not as opposite; they may or may not two may at times appear to be the same. However, even when they do, it would still be the figure that is related to rather than the facts themselves. route as discussed by De Certeau [17]. The discussion above has aimed at a further extension, which is avoiding a projected possibility, and the bases for such a projection. This is shown as simplified types of avoidance in figure 3 .
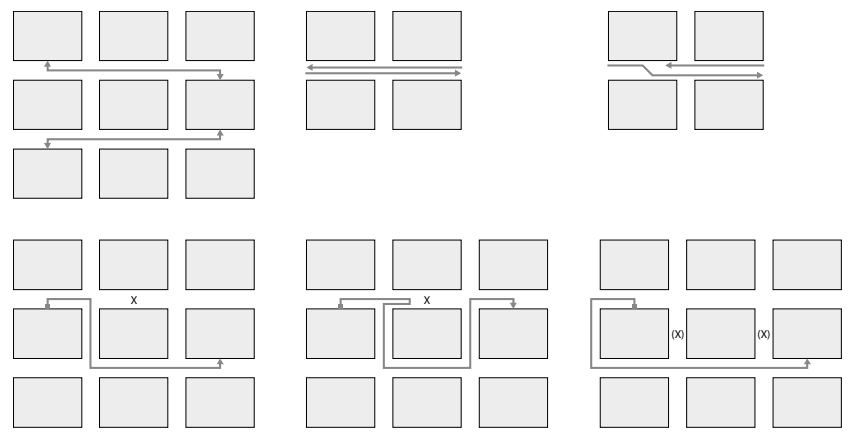

Figure 3. Types of avoidance. The top left illustrates 'programmed avoidance' - two people moving between two places are unlikely to meet because of how their origins, destinations, and paths are arranged. Top centre shows the avoidance discussed by Simmel and Benjamin of people not recognising one another as they physically meet in, for instance, a street. Top right illustrates the avoidance of changing sides of a street to avoid an encounter. Bottom left extends this to the choice between alternate, otherwise equally close, routes to avoid an encounter. Bottom centre illustrates taking a longer route in order to avoid an encounter. Bottom right, finally, illustrates the choice to take a longer route based on possible, projected encounters. While the first can operate largely on hands-on information, the last requires the projected activity of others to be enacted.

In this tactical or strategic avoidance, memory, myth and metonymy play crucial roles as avoidance depends on projecting and assuming the intentions and actions of the other. While arguably a part of every action, this becomes especially important in the form discussed here since it ultimately depends on assumptions. I can here remind of the different forms of the game of hide-and-seek: in similar ways, avoidance as a tactical or strategic component of action relates to memories, myths and metonymies to facilitate relations to the others, the generic other, the Other, that is, to the activities, events, risks or potentials one wishes to avoid. It is important to again stress that avoidance is not by necessity avoidance of something 'bad', but rather from something not in alignment with the current mood, intentions, plans or preferences. Or simply put; some things we do together, some we don't [35]. On a first date one might avoid places where one is most likely to encounter friends, acquaintances or relatives [47]; one may avoid most everyone else when going for a quiet picnic; one may avoid specific others when buying a gift; or one may simply not be in the mood, be in a rush, or have any other everyday reason to prefer to not meet someone and risk being rude. It can be a question of marking identity, as Zukin and Kosta discusses regarding 'off-broadway' locations of certain ranges of shops [39]. It allows co-existence of difference, and facilitates emergences of subcultures, but also risks maintaining or even strengthening segregation, prejudices, social difference and distrust. Two persons, or groups, avoiding one another 
would be caught in a dynamic, ever-renegotiated game of hide-and-seek - or perhaps hide-and-hide - not entirely different from Goffman's [18] discussion on civil inattention, but on a level related to spatial configuration and chance of co-presence rather than at face-value of being in the same space, which may result in temporarily stable patterns or a constantly shifting range of actions and states.

At this point, it is worth returning to the contribution of the discussion to our understanding of social actions and structures in general, which I will argue is twofold. One is the embededness of actions in a temporal and spatial extension that includes projections, plans and assumptions. That is, it is not only avoidance that depends on memory, myth, and metonymy but arguably a wide set of actions, if not most. The other is the component of avoidance itself in structuring action and thereby, performatively, social structures. Who, what, when, and where we chose to avoid, or which is arranged for to be avoided, becomes powerfully communicating and structuring choices. Rather than considering specific acts of avoidance, which has been used here so as to move the discussion onwards, it is also clear how many examples provided depends on both desired and undesired outcomes. ${ }^{17}$ For instance, in the referred example from Nadja, the protagonist and Nadja does not only avoid places where they may meet friends or acquaintances, but also seek out a situation in which they wish to spend their evening.

\section{Spatial Conditions of Avoidance}

Before concluding, I will return to some of the spatial conditions of 'avoidance'. I will do so only briefly as deeper research on such conditions remain to be conducted. To start, it can be noted that spatial arrangements into series, tree, and network as discussed by Foucault $[40]^{18}$, or the plans of Palazzo Antonini (Andrea Palladio, 1556) and The Red House (Philip Webb, 1859) in Robin Evans' Figures, Doors and Passages [31] are radically differently appropriate for variants of the game of 'hide-and-seek'. A series, for all practical intents and purposes, is not meaningful for the game as it would simply amount to how far the seeker would have to go to find the ones hiding. A tree, similarly to The Red House, allows for slightly better possibilities, as hiders can potentially reposition themselves while the seeker searches a branch, leaving the spine

17 Rather than a binary either/relation between avoided and desired, it is fruitful to consider social actions as including positive and negative possibilities weighed against one another. This could be compared to the Aristotelean 'logical opposition' and 'real opposition' [57], where the former is binary and opposing forces constitutes the latter. Any position (or action) in the latter is the result of the forces that affect it, and the forces are embedded in the position or action, whereas in the former they are neutralized into the position (action).

18 These three relational concepts appear in a less clear form in The Social Logic of Space through different conceptual plans of buildings, and have since been refined to more closely correspond to Foucault's concepts by Bafna [58]. While used to provide a conceptual introduction of spatial configuration and how closely it is tied to culture and ideals. In the research field 'space syntax' at large, however, focus has rather been elsewhere than on this relation, even if works like those of Markus [28], Hanson [30] and Psarra [59] amongst others develops on it extensively. unsupervised. A network, finally, or the Palazzo Antonini, sets the dynamics further in motion: allowing for any action to be countered, the game of anticipation and tactics become richer.

At first it seems that the more distributed a configuration, the more it allows avoidance. However, one should not too readily accept this, as it can be further said that the more distributed a system, the less predictive it becomes: while 'I' do not have to pass through any specific spaces, neither does anyone else. Offering more possibilities of avoidance, a more distributed system further requires more assumptions of the actions of that which one intends to avoid. As noted by Hillier and Hanson [1] there comes a point where a game of hide-and-seek fails because the environment is too rich with alternatives. Different activities would relate differently to configuration from this point of view, further related to whether one acts in the whole or in sub-sections of the system. As Hanson [30] notes, while Hardwick Hall is hierarchically structured globally, "[t]he deep, ringy structure which lies beyond Bess's private, separate and protected sub-complex is well-nigh impossible to police" (p. 193) but furthermore, that "this may be unnecessary and even undesirable where the occupants have shared attitudes and values."

This indicates how different configurations of space allow differently for avoidance, and furthermore illustrates the relation between avoidance and freedom as an inverse of control (c.f. [28]). This does not mean that avoidance and freedom are interchangeable concepts - far from it. It does, however, suggest that certain practices of avoidance and freedom are similarly conditioned, and that in some cases avoidance can be an enactment of freedom.

\section{Conclusions: Avoidance and the Performative Dynamics of Social Structures}

To conclude, I will reiterate a few things from above, and return to the question of why 'avoidance'. I will begin where I began the essay: I believe that a study of avoidance leads to a range of questions that need to be answered regarding relations between activity and space, which can potentially be disregarded in a discussion of presences, interactions, movement or encounters. These questions provide a richer understanding of social structuring processes and their interplay with space. While many of the immediate examples that may come to mind as we speak of avoidance may be troublesome, part of the argument is that this assumption often experienced as a gut-reaction needs to be avoided. Both because, as Mary McLeod notes in 'Everyday and "Other" Spaces' [60], the concept of heterotopias [40] can also be used to describe everyday spaces and activities of regular life, if not for all then many or most, some of which may form pockets of freedom in the durée of daily life (c.f. De Certeau's [17] 'network of anti 
discipline', p. xv). Indeed, heterotopias as being places outside of hegemonic structures can offer freedoms from the same - keeping in mind the way in which those that avoid or are avoided can be dislocated, displaced or excluded with various direct social, spatial and material implications. Various forms of avoidance form important parts of urban life allowing individuals or groups who may have conflicting interests to live together either by direct, shared 'inattention' as they meet [18], or by shared knowledge allowing stressful meetings to be avoided. Such inattention and avoidance is dependent on constant ongoing negotiation and interaction to be maintained.

Still, while the various forms of heterotopias possible to relate to avoidance form important pieces of the argument, the main form of avoidance discussed in this essay should be related to De Certeau's spatial tactics as played out in collective socio-cultural spatial dynamics. In part a form of what Zukin [61] labels the 'negotiation of public culture', but not restricted to Zukin's term 'public', but as part of the structuring of 'the social' as a whole as a performative, enacted system $[12,23]$.

The point here is not the good or bad of avoidance but that it is and will remain enacted - and that through its enactments it generates structures of actions, perceptions, reactions and habits that form structures that defy any reading that does not recognise memory, anticipation and projection as well as differentiation and avoidance as integral aspects of behaviour.

To conclude, then, a few notes can first be made in respect to the discussion held throughout this paper:

First, it can be noted how the constantly ongoing sense-making, othering, subjectification, and negotiated actions and relations highlighted in this essay form integrated parts of any action and activity. That is, it is clear that any social action depends on memories, mythologies, and metonymies in addition to material and functional aspects, where the latter could be argued to be included in the former. Focusing on avoidance has allowed and necessitated these to be addressed in-depth.

Second, it can be noted how this leads to the enactment of perceptions and structures related to this, which performatively formulates, defines, and describes social and cultural relations through how it participates in structuring encounters and avoidances; this takes place both directly in who meets whom, and indirectly in who means to meet or avoid whom, and who is expected to meet or avoid whom, propagated throughout society by means of its own enactment, with implications for just what co-absence could be as different from just 'empty' or 'unused' space. ${ }^{19}$ That

19 While for the one unknowing encountering the space they may seem similar, the latter simply depends on lack of presence whereas the former is always already socially and politically charged. Co-absence in this sense may be one of the clearest expressions of the structuring effects of avoidance as examples of their dependency on assumption, prejudice, and projection. That is, while none of those avoiding a space know if the other is there, the reason they are not there is that the other could be, and as a result, due to assumptions of one another's behaviour, several parties avoid to enter, go to, or pass through a certain space, for a particular period, or a particular time, is, myths, memories and metonymies are reified through their direct or indirect enactment as temporary (approximations of) truths.

Third, these form complex patterns of action that in part formulate the basis for themselves that are constantly renegotiated on several levels, as the encounters that do happen can challenge, confirm, twist, or alter perceptions, attitudes and expectations directly or over time - dependent on the way they are interpreted, which is interdependent with perceptions and ideas, processes of subjectification and othering as discussed here as well as ideas of societal structures and processes.

These three notes are concerned with the performative, that is, the way structures emerge through their enactment. More concretely, the discussion in this essay points to the importance of projective relations - that is, how pasts and futures form components of concurrent actions, and thereby participates in social structuring. In this, 'avoidance' becomes a special case in discussing the temporal and spatial embededness of the 'here and now', not only in what happens before, after and elsewhere, but what could take place or might have taken place. It also challenges notions of actions as a result of preference and rather suggests actions to depend on a complex set of positives and negatives where arguably all actions carry proportions of both, but where which dominates may differ radically for ostensibly similar behaviour. That is, am I on this street because I want to be, or because I do not wish to be on another street? This, arguably, is not a binary or logical opposition of either/or, but rather a saturated relation of both/and.

For an architecture or urban theory concerned with the 'social' in any of its broader meanings, these must be central concerns, and discussions of how to provide lively places, the interaction processes in these, the creation of community, and similar concerns can only be considered as insufficient. They can be further questioned as ideals, at least as generic ideals, as they risk leading to a consistent spreading out of a mean, acceptable public culture that is tolerable and tolerant only to what does not unduly disturb it, and where other preferences will have to be sorted out as oddities or anomalies. Social tension and differences, to an extent worked out through differentiation and avoidance, to an extent created and maintained by the same, arguably become what allows differences to co-exist. But it also leads to questions of how we interpret individual or collective action, and how social structures emerge from and relate to these actions.

\section{REFERENCES}

[1] B. Hillier and J. Hanson. The Social Logic of Space, Cambridge University Press, Cambridge (UK), 1984

or more or less perpetually. 
[2] B. Latour. Reassembling the Social: An introduction to actor-network theory, Oxford University Press, New York, 2005

[3] K. Day. Strangers in the Night: Women's fear of sexual assault on urban college campuses, Journal of Architecture and Planning Research, Vol. 16, No. 49, 289-312, 1999

[4] J. Hollander. Vulnerability and Dangerousness: The construction of Gender through Conversations about Violence, Gender and Society, Vol. 15, No. 1, 83-109, 2001

[5] G. Valentine. Images of Danger: Women's sources of information about the spatial distribution of male violence, Area, Vol. 24, No. 1, 22-29, 1992

[6] L. Logan. Fear of Violence and Street Harassment: Accountability at the intersections, Kansas State University, Manhattan (Kansas), 2013

[7] P. L. Brantingham and P. J. Brantingham. Nodes, Paths and Edges: Considerations on the complexity of crime and the physical environment, Journal of Environmental Psychology, Vol. 13, 3-28, 1993

[8] R. Samuels. After-Dark Design, Night Animation, and Interpersonal Interaction: Towards a community-security paradigm, Journal of Architectural and Planning Research, Vol. 22, No. 4, 305-318, 2005

[9] V. Ceccato (ed.). The urban fabric of crime and fear, Springer, Dordrecht, 2012

[10] V. Ceccato. Safety on the move: Crime and perceived safety in transit environments, Security Journal, Vol. 27, No. (2), 127-131, 2014

[11] F. Tonkiss. Space, the City and Social Theory: Social relations and urban forms, Polity Press, Cambridge, 2005

[12] M. Rose. The seductions of resistance: power, politics, and a performative style of systems, Environment and Planning D, Vol. 20, 383-400, 2002

[13] G. Simmel. The Metropolis and Mental Life (trans. H. Gerth), in K. H. Wolff (ed.), The Sociology of Georg Simmel, Free Press, Glencoe, 409-424, 1950 (1903)

[14] W. Benjamin. The Arcades Project (trans. H. Eiland and K. McLaughlin), Harvard University Press, London, 1999

[15] J. P. Williams. Subcultural Theory: Traditions and concepts, Camrbidge (UK), Polity Press, 2011

[16] K. Bonnevier. Behind Straight Curtains: Towards a queer feminist theory of architecture, Axl Books, Stockholm, 2007

[17] M. De Certeau. The Practice of Everyday Life (trans. S. Rendall), University of California Press, London, 1984

[18] E. Goffman. Behavior in Public Places, Free Press of Glencoe, New York, 1963

[19] L. Lofland. A World of Strangers: Order and Action in Urban Public Space, Basic Books, New York, 1973

[20] J.-F. Augoyard. Step by Step: Everyday walks in a French urban housing project (trans. D. A. Curtis), University of Minnesota Press, Minneapolis, 1979

[21] R. Barthes, Mythologies (trans. J. Cape and S. Reynolds), Vintage, London, 2009 (1957)
[22] P. Ricoeur. Hermeneutics and the Human Sciences (trans. J. B. Thompson), Cambridge University Press, Cambridge (UK), 1981

[23] J. Butler. Gender Trouble, Routledge, New York, 1999

[24] D. Pop. "Hide and Seek" - Expressing the Behavioural Norms of the Group and the Environment. Acta Technica Napocensis: Civil Engineering \& Architecture, Vol. 56, No. 3 , 228-235, 2013

[25] M. Foucault, Discipline and Punish: the birth of the prison, Penguin, Harmondsworth 1991

[26] T. Bennett. The Birth of the Museum London. Routledge, London, 1995

[27] B. Colomina. Privacy and Publicity: Modern Architecture as Mass Media, MIT Press, Cambridge (MA), 1996

[28] T. A. Markus. Buildings \& Power: Freedom \& Control in the Origin of Modern Building Types, Routledge, London, 1993

[29] J. Hanson. The architecture of justice: iconography and space configuration in the English law court building, Architectural Research Quarterly, Vol. 1, No. 4, 50-59, 1996

[30] J. Hanson. Decoding Homes and Houses, Cambridge University Press, Cambridge (UK), 1998

[31] R. Evans. Figures, Doors and Passages, Architectural Design Vol. 48, 267-278, 1978

[32] P. Emmons. Intimate Circulations: Representing Flows in House and City, AA Files Vol. 51, 48-57, 2004

[33] P. Steadman. Building Types and Built Forms, Matador, Leicestershire, 2014

[34] J. W. R. Whitehand and C. M. H. Carr. Twentieth Century Suburbs: A morphological approach, Routledge, London, 2001

[35] D. Koch. Structuring Fashion: Department stores as situating spatial practice, Axl Books, Stockholm, 2007

[36] C. R. V. Tandy. (1967). The isovist method of landscape survey, in H. C. Murray (ed.), Methods of Landscape Analysis, Landscape Research Group, London, 1967

[37] M. L. Benedikt. To take hold of space: isovists and isovist fields, Environment and Planning B, Vol. 6, 47- 65, 1979

[38] A. Turner and A. Penn. Making isovists syntactic: isovist integration analysis. Paper presented at the 2nd International Symposium on Space Syntax, Universidad de Brasilia, Brazil, April 1999

[39] S. Zukin and E. Kosta. Bourdieu Off-Broadway: Managing distinction on a shopping block in the East Village, City \& Community, Vol. 3, No. 2, 101-114, 2004

[40] M. Foucault. Of Other Spaces: Utopias and Heterotopias, in N. Leach (ed.), Rethinking Architecture: a reader in cultural theory, Routledge, London, 350-356, 1997 (1985)

[41] A. Legeby. From Housing Segregation to Integration in Public Space: A space syntax approach applied on the City of Södertälje, Journal of Space Syntax, Vol. 1, No. 1, 92-107

[42] J. Madge. Type at the origin of architectural form, The Journal of Architecture, Vol. 12, No. 1, 1-34, 2007 
[43] D. Koch. Changing Building Typologies: The typological question and the formal basis of architecture, Journal of Space Syntax, Vol. 5, No. 2, 168-169, 2014

[44] A. Gramsci. Selections from the Prison Notebooks of Antonio Gramsci, Lawrence and Wishart, London, 1971

[45] H. Ferguson. Self-identity and everyday life, Routledge, Oxon, 2009

[46] V. Netto. Practice, space, and the duality of meaning, Environment and Planning D, Vol. 26, 359-379, 2008

[47] A. Breton. Nadja (trans. R. Howard), Grove Press, New York, 2000 (1928)

[48] P. Bourdieu. Distinction: a social critique on the judgement of taste (trans. R. Nice), Routledge, London, 1984 (1979)

[49] S. de Beauvoir. The Second Sex (trans. H. M. Parshley), Vintage, London, 1997 (1949)

[50] E. Said. Orientalism, Pantheon Books, New York, 1978

[51] V. Burgin. In/different spaces: place and memory in visual culture, University of California Press, Berkeley, 1996

[52] N. Kaye. Site Specific Art: Performance, place and documentation, Taylor \& Francis, London, 2000

[53] M. J. Biondolilo and D. B. Pillemer. Using Memories to
Motivate Future Behaviour: An experimental exercise intervention, Memory, Vol. 23, No. 3, 390-402, 2015

[54] A. E. Wilson and M. Ross. The identity function of autobiographical memory: Time is on our side, Memory, Vol. 11, No. 2, 137-149, 2003

[55] L. Lofland. The Public Realm: Exploring the city's quintessential social theory, Aldine Transaction, London, 2009 (1998)

[56] S. Žižek. The Pervert's Guide to Ideology, Zeitgeist Films / British Film Institute / Film4, 2012

[57] G. Chatelet. Figuring Space: philosophy, mathematics, and physics (trans. R. Shore and M. Zagha), Dordrecht: Kluwer, 2000

[58] S. Bafna. Space Syntax: A brief introduction to its logic and analytical techniques, Environment and Behavior, Vol. 35, No. 1, 17-29

[59] S. Psarra. Architecture and Narrative: The Formation of Space and Cultural Meaning, Routledge, London, 2009

[60] M. McLeod. 'Everyday and "Other" Spaces', in D. Coleman, E. Danze, and C. Henderson, Architecture and Feminism, Princeton Architectural Press, New York, 1-37, 1996

[61] S. Zukin. The Cultures of Cities, Blackwell, Oxford, 1995 